\title{
La gobernabilidad de los servicios de agua y saneamiento y los conflictos por el agua en América Latina
}

Melisa Orta ${ }^{1}$

Universidad Nacional de Rosario

\section{Artículo científico}

Material original autorizado para su primera publicación en el Journal de Ciencias Sociales, Revista Académica de la Facultad de Ciencias Sociales de la Universidad de Palermo.

Recepción: 31-01-2018

Aceptación: 03-05-2018

Resumen: En las últimas décadas, tuvo lugar un incremento progresivo de la importancia que ha adquirido a nivel internacional el problema de las desigualdades existentes en el acceso a los servicios vitales de agua y saneamiento. A pesar de que se han fijado metas y objetivos que propendieron a su resolución, las desigualdades aún persisten e incluso, en algunos aspectos, se han profundizado. En este trabajo nos proponemos realizar un repaso de algunas de las dimensiones de análisis de este problema abordadas en la Ciencia Política, a partir de tres conceptos considerados nodales para estudiar la persistencia y la profundización del problema de la desigualdad en el acceso a estos servicios públicos. Estos son: gobernabilidad, capacidades estatales y conflictos hídricos. El objetivo es identificar cuáles son los desafíos y obstáculos más importantes que analiza la bibliografía especializada para avanzar en la democratización del acceso a los servicios de agua y saneamiento seguros. A este efecto, tratamos de identificar factores clave para poder formularnos preguntas que nos permitan estudiar cuáles son los obstáculos y desafíos para avanzar hacia la democratización en el acceso a estos servicios esenciales en la provincia de Santa Fe (Argentina). Las conclusiones presentan una síntesis de los argumentos principales.

Palabras clave: agua y saneamiento; desigualdad social; gobernabilidad; conflictos hídricos.

\section{Governability of Water and Sanitation Services and Conflicts Over Water in Latin} America

\footnotetext{
${ }^{1}$ Centro de Estudios Interdisciplinarios (CEI), Universidad Nacional de Rosario (UNR) / Consejo Nacional de Investigaciones Científicas y Técnicas (CONICET)Centro de Estudios Interdisciplinarios de la Universidad Nacional de Rosario. Correo electrónico: melisaorta@gmail.com
} 
Abstract: In recent decades, there has been a progressive increase on the importance of considering the problem of real inequalities to have access to water and sanitation services at an international level. Although certain goals and objectives were set in order to handle this matter, the inequalities not only persist but also have deepened. In this paper we propose to review the different dimensions of analysis addressed by the Political Science, based on three concepts considered nodal for the study of the persistence and deepening of the problem of inequality in access to these public services. These are: governability, state capacities and hydrological conflicts. The objective is to identify which are the most important challenges to be faced and obstacles to be overcome, analysed by the specialized bibliography, to advance towards the democratization of the access to safe water and sanitation. To this effect, it is our intention identify key factors that will allow to ask questions us to study what the obstacles and challenges are to move towards democratization in the access to these essential services in the province of Santa Fe (Argentina). The conclusions present a synthesis of the main arguments.

Key words: water and sanitation; social inequality; governance; hydrological conflicts.

\section{Introducción}

El presente trabajo tiene como objetivo presentar algunos temas clave que se destacan en la literatura sobre los desafíos y obstáculos existentes para enfrentar la persistencia de desigualdades en el acceso a los servicios básicos y esenciales de agua y saneamiento en América Latina².

En primer lugar, nos interesa dar cuenta del grado de importancia que ha adquirido esta problemática a nivel internacional y de por qué se asume como fundamental el abordaje de las dimensiones políticas y sociales, tanto para identificar las causas que originan las desigualdades como para poder desarrollar soluciones efectivas.

En segundo lugar, daremos cuenta de algunos de los estudios que se han desarrollado desde la Ciencia Política partiendo de tres conceptos considerados nodales para abordar el problema de la desigualdad en el acceso a los servicios básicos. Estos son: gobernabilidad, capacidades estatales y conflictos por el agua.

En tercer lugar, presentaremos brevemente una descripción sobre el estado actual de la gestión de los SAS en la Provincia de Santa Fe (Argentina) y qué aspectos de la bibliografía explorada a lo largo del trabajo nos permiten desarrollar interrogantes para conocer cómo se

\footnotetext{
2 Hacemos referencia al servicio de agua potable y desecho de residuos cloacales por red, que, en el caso específico de la provincia de Santa Fe, son provistos por una empresa perteneciente al Estado provincial, por los gobiernos locales o por cooperativas.
} 
gobiernan los servicios de agua y saneamiento y para identificar cuáles serían los desafíos y obstáculos que debe enfrentar la provincia para avanzar en la democratización del acceso a estos servicios.

\section{Las desigualdades en el acceso a los servicios de agua y saneamiento como un}

\section{problema social y político}

Para dar inicio a este trabajo, nos interesa aclarar que partimos de una concepción marxista para poder entender y analizar cuáles son las causas que producen y reproducen las desigualdades sociales. Para Marx, las desigualdades son consecuencia de procesos sociales históricamente específicos relativos al modo de producción dominante ${ }^{3}$. Una de las formas predominantes en la fase neoliberal por la cual el capitalismo se reproduce y supera sus crisis de sobreacumulación de capital y fuerza de trabajo, siguiendo a Harvey (2005), es a través de la acumulación por desposesión. Esta se caracteriza por procesos tales como los ataques llevados a cabo por fondos financieros contra la soberanía económica de los países (fuga de capitales, crisis de liquidez, devaluación); la proliferación de la degradación ambiental; la corporativización y privatización de activos previamente públicos; la ola de privatización de las fuentes de agua, de los servicios de agua y saneamiento y otros servicios públicos; la vuelta al dominio privado de derechos de propiedad común (el derecho a una pensión estatal, al bienestar, al sistema de salud nacional), entre otros (Harvey, 2004, pp. 7475). Estos procesos profundizan las desigualdades sociales, aumentando la brecha entre ricos y pobres y deteriorando las condiciones de vida de las clases desposeídas.

Estas desigualdades, se manifiestan también en el nivel de acceso de la población a los servicios vitales de agua y saneamiento. En el año 2002 en América Latina, según el Programa Conjunto de Monitoreo del Abastecimiento de Agua y Saneamiento (PCM) a cargo de la Organización Mundial de la Salud (OMS) y el Fondo de las Naciones Unidas para la Infancia (UNICEF, por sus siglas en inglés), un $11 \%$ de la población de América Latina y el Caribe (60 millones de personas) carecían de acceso a fuentes de agua mejorada y un $25 \%$ de la población (137 millones de personas) no tenía acceso a instalaciones de saneamiento mejoradas $^{4}$ (OMS y UNICEF, 2004). La falta de acceso a estos servicios esenciales acarrea serios problemas para la salud y deteriora la calidad de vida de las personas afectadas.

Ante esta situación preocupante, los países miembros de la Organización de Naciones Unidas (ONU) asumieron diferentes compromisos internacionales para enfrentar el

\footnotetext{
3 Para profundizar en las distintas definiciones existentes en las Ciencias Sociales sobre el concepto de desigualdad social se puede ver el trabajo realizado por Castro (2016a).

${ }^{4}$ EI PCM conceptualiza como fuente de agua mejorada a aquella que cumple criterios específicos relativos a la protección de la fuente y al tratamiento del agua, pero que no necesariamente proporcionan agua salubre a las personas (OMS y UNICEF, 2007, p. 4).
} 
desafío de reducir las desigualdades en el acceso a los Servicios de Agua y Saneamiento (SAS). De estos compromisos, cabe resaltar el de los Objetivos de Desarrollo del Milenio (ODM) en al año 2000, la declaración del acceso al agua y saneamiento como un derecho humano en 2010 y el compromiso de los Objetivos de Desarrollo Sostenible (ODS) en 2015.

El compromiso de los ODM fue asumido en el año 2000, cuando representantes de 189 países de todo el mundo se reunieron en la Sede de la ONU en Nueva York y acordaron el establecimiento de objetivos y metas para combatir la pobreza, el hambre, el analfabetismo, la discriminación contra la mujer, las enfermedades y la degradación del ambiente (ONU, 2000). Entre estos objetivos, el que estaba relacionado de forma directa con los SAS proponía reducir a la mitad, para el año 2015, el porcentaje de la población mundial que en 1990 no tenían acceso sostenible al agua potable y a servicios básicos de saneamiento ${ }^{5}$. Esto implicaba, para la región de América Latina y el Caribe, pasar de un 85\% en 1990 de la población con acceso a fuentes mejoradas de agua a un 92,5\% en 2015. Respecto al acceso a instalaciones mejoradas de saneamiento, implicaba avanzar desde un 69\% en 1990 a un $84 \%$ en 2015.

Con el objetivo de profundizar la importancia que tiene a nivel internacional la problemática de acceso a estos servicios esenciales, la Asamblea General de la ONU, en julio de 2010, estableció que el acceso seguro al agua potable salubre y al saneamiento constituyen un derecho humano fundamental para el completo disfrute de la vida y de todos los demás derechos humanos (Resolución 64/292 de 2010). El reconocimiento del Derecho Humano al Agua y Saneamiento (DHAS) implica que cada persona, sin importar raza, sexo, nacionalidad o condición social, puede demandar el acceso a los mismos, ya que, se los considera como imprescindibles para lograr un nivel de vida adecuado ${ }^{6}$.

Cumplido el plazo de los ODM, en 2015 el PCM publicó una serie de informes en los cuales se analizó el avance mundial para alcanzar las metas y objetivos propuestos. Los informes celebran el hecho de que nominalmente se habría logrado el objetivo de reducir a la mitad la proporción de la población mundial que no tenía acceso a fuentes de agua "mejoradas". Sin embargo, 45 países -el 23\% por ciento de un total de 192 países- no habían alcanzado el objetivo. Con relación al saneamiento, la situación es aún más grave ya que sólo habían alcanzado el objetivo 95 países (49\% del total de 192). Con relación a América Latina, la región en su conjunto logró superar la meta referida al acceso a fuentes mejoradas de agua al pasar de un 85\% de cobertura en 1990 a un 95\% en 2015. Con relación a la meta de saneamiento, se estuvo próximo a alcanzarla, ya que un $83 \%$ de población tuvo acceso a

\footnotetext{
5 Si bien la formulación de los ODM data de 2000, las bases de la mayor parte de las metas incluidas en dichos ODM se establecieron ya en 1990.

${ }^{6}$ Este reconocimiento se había realizado en forma implícita en diversos acuerdos internacionales precedentes (Cfr. Mora y Dubois, 2015, p. 9).
} 
instalaciones mejoradas. No obstante, 11 países no habían logrado alcanzar la meta para el agua y 19 países no lograron la meta de saneamiento (OMS y UNICEF, 2015). Cabe aclarar que el hecho de que más personas tengan acceso a fuentes e instalaciones "mejoradas" no significa necesariamente que reciben agua adecuada para consumo humano o accedan a estructuras de saneamiento libres de contaminación (OMS y UNICEF, 2004, p. 23). Además, aún persisten profundas desigualdades geográficas, socioculturales, económicas y entre regiones urbanas y rurales que, en algunos casos, se han agudizado. Según uno de los informes elaborados por el PCM, la gran mayoría de las personas que no tienen acceso a servicios de saneamiento son las personas más pobres que viven en las áreas rurales o en las zonas marginales de las grandes ciudades (OMS y UNICEF, 2014, citado por Castro, 2016b).

En el año 2015 se declararon los Objetivos de Desarrollo Sostenible (ODS), que retoman y profundizan los ODM y se proponen conseguir lo que estos no lograron. El período para alcanzarlos comprende los años 2016-2030. El objetivo relacionado con el tema que nos ocupa es el número 6, que se propone "Garantizar la disponibilidad y la gestión sostenible del agua y el saneamiento para todos" (ONU, 2015, p. 21). Cómo se puede ver, este objetivo es más ambicioso que las metas que se contemplaban en la anterior agenda de desarrollo debido a que se propone alcanzar la universalización del acceso a los SAS. Así mismo, aborda la problemática considerando todos los componentes del ciclo, incluyendo la gestión del agua, de las aguas residuales y de los recursos de los ecosistemas (ONU, 2015, p. 21).

Ahora bien, una literatura creciente considera que los desafíos para poder alcanzar la universalización del acceso al agua y saneamiento seguros y garantizar el DHAS no son sólo de carácter natural o técnico, ni siquiera económico. Esto quiere decir que no es sólo la disponibilidad natural de agua, el acceso a soluciones tecnológicas, ni el nivel de disponibilidad de recursos económicos lo que determina que se efectivice el acceso a estos servicios. Sino que es central, también, el modo en cómo se gestionan estos servicios y las relaciones sociales de poder que se constituyen y posibilitan o dificultan la efectividad de las políticas públicas que tienden a la concreción de estos derechos (Castro, 2007; Swyngedouw, 2004). Es por esto, que nos interesa destacar algunas de las líneas de investigación que se han desarrollado en el campo de la Ciencia Política en este sentido.

Podemos decir que los temas principales que se han abordado desde la Ciencia Política con relación a la problemática de las desigualdades en el acceso a los SAS están relacionados con la gobernabilidad y las capacidades estatales para gestionar los servicios, por un lado y, por el otro, con el estudio de los conflictos y luchas sociales por el agua. Ambos temas, como veremos más adelante, están estrechamente relacionados. A continuación, 
destacaremos algunos aspectos centrales que surgen de las investigaciones recientes sobre el tema y los principales conceptos y dimensiones que abordan.

\section{Gobernabilidad de los servicios de agua y saneamiento}

En relación con el tema de la gobernabilidad de los servicios de agua y saneamiento, nos interesa comenzar dando cuenta del estudio llevado a cabo por Castro (2011). El autor investigó las distintas lógicas de gobernabilidad de los SAS imperantes a lo largo de la historia, su relación con la concepción de los derechos de ciudadanía y sus implicancias para la democratización en el acceso y gestión de estos servicios. En este sentido, define a la gobernabilidad como la forma en que se establecen relaciones sociales de poder entre los distintos actores sociales y políticos involucrados y el modo en que cierta visión o concepción sobre el recurso hídrico logra legitimarse en cada momento histórico. De esta manera, Castro considera que la cuestión de la gobernabilidad de los servicios esenciales de agua y saneamiento se puede estudiar considerando los interrogantes siguientes:

¿Cuáles son los mecanismos para la designación de los funcionarios y expertos a cargo de la gestión de los servicios de agua y saneamiento? ¿Quién los designa y con qué criterios? ¿Cómo se definen las metas que la sociedad debe fijarse con relación a la gestión de los servicios esenciales? ¿Qué valores e intereses materiales se reflejan en dichas metas? ¿A quién pertenecen los valores e intereses materiales en función de los cuales se fijan dichas metas? [...] ¿Qué mecanismos tienen los ciudadanos y las ciudadanas para ejercer control sobre los actores e instituciones que están a cargo de estas decisiones y de su implementación? (Castro, 2011, p. 72).

A partir de encontrar respuestas empíricas a estos interrogantes, el autor realiza un análisis de las tendencias centrales que caracterizaron los sistemas de gestión del agua a lo largo de su desarrollo histórico. Desde el inicio de estos servicios hasta mediados del siglo XIX predominó un modelo de tipo privatista, donde los sistemas de provisión de agua, en las principales ciudades de Europa y Estados Unidos, estaban a cargo de compañías privadas y abastecían del servicio solamente a los sectores de mayor poder adquisitivo. Los principios que caracterizan a este modelo son: (a) el agua potable es concebida como una mercancía, un objeto de propiedad privada cuyo intercambio debe ocurrir de acuerdo con las reglas del mercado y sin interferencia del Estado, las personas son responsables de comprar estos servicios en forma privada; (b) en cuanto al criterio para expandir los servicios prima el principio de exclusión, el cual determina que el acceso al agua domiciliaria es considerado un bien privado, disponible solamente para quienes pueden pagar por él y se admite el corte del servicio a quienes no pagan y (c) con relación al proceso de toma de decisiones tiene protagonismo el sector privado, excluyendo la participación ciudadana (Castro, 2011, pp. 6769). 
Una segunda etapa comienza a finales del Siglo XIX y principios del Siglo XX, cuando la intensificación del proceso de industrialización generó el crecimiento exponencial de la población en las ciudades. Esto hizo que la lógica privatizadora fuera insuficiente para responder a las demandas de urbanización de estos nuevos sectores que luchaban por la ampliación de sus derechos. A partir de ese momento, comenzó a generarse un proceso de estatización, el Estado comenzó a crear empresas que tenían a su cargo la provisión de estos servicios. Los principios que caracterizan a la lógica estatista racional administrativa son: (a) la provisión de los servicios de agua y saneamiento constituye un servicio público de acceso universal, independiente de la capacidad de pago de los individuos y las familias; (b) el acceso a este servicio público constituye una función indelegable del Estado, el cual debe asegurar su prestación en forma regular, uniforme, general y continua y (c) en cuanto a la gestión de los SAS, restringe la participación en la toma de decisiones a los políticos profesionales y a los expertos técnicos. Las decisiones se toman de modo verticalista, sin la apertura de instancias de participación ciudadana. En algunos casos, se identificó la existencia de instancias de participación, pero sus decisiones eran de carácter no vinculante. Este proceso fue acompañado por la inclusión de los derechos sociales, que concebían que todo habitante de una comunidad tenía derecho a acceder a ciertos bienes básicos y comunes tales como el derecho a una vivienda, salud, educación y saneamiento (Castro, 2011, pp. 69-72; Groisman, 1995, citado por Tobías, 2014, p. 24).

A principios de la década de 1990, se puede identificar una nueva etapa para la región de América Latina, iniciada por las reformas neoliberales impulsadas por el Consenso de Washington. Durante la misma, muchas de las empresas que eran de gestión público-estatal pasaron a ser concesionadas o vendidas a consorcios privados. En esta etapa, cobran vigencia nuevamente los principios privatistas que mencionamos anteriormente (mercantilización, exclusión, rentabilidad). El retorno del privatismo puso en cuestión el principio de universalidad que había formado parte del consenso predominante durante la mayor parte del siglo XX, lo cual tuvo consecuencias en el aumento y profundización de las desigualdades sociales en cuanto al acceso a estos servicios vitales (Swyngedouw, 2005; Goldman, 2007; Spronk, 2007).

A inicios del siglo $X X I$, asistimos a un proceso de reestatización de las empresas prestadoras de los servicios. Sin embargo, el regreso de la lógica de gestión estatista no se encuentra exento de la incorporación de principios propios de modelo privatista. Esta situación ha sido caracterizada por teóricos especialistas en el análisis de cómo se gestionan los servicios públicos bajo el concepto de corporatización. Las entidades corporatizadas son de propiedad y gestión estatales, pero poseen un grado de autonomía respecto a los funcionarios políticos. Asimismo, pueden incorporar en su funcionamiento principios mercantilistas tales como: la fijación de tarifas que reflejen los costos, cortes generalizados 
del servicio de agua a familias con bajos ingresos, énfasis en mejorar la eficiencia operativa y la disciplina fiscal, promoción de la autofinanciación de los servicios eliminando progresivamente los subsidios, entre otros (McDonald, 2015, pp. 26-27).

Los estudios reseñados hasta aquí nos permiten contar con un marco general de cómo se desarrollaron estos servicios y las implicancias de las distintas lógicas de gestión imperantes sobre el grado de democratización en su gestión y acceso. Ahora bien, nos interesa focalizarnos en trabajos que profundizaron en cada una de estas etapas en los procesos que tuvieron lugar en América Latina y específicamente en Argentina.

\subsection{Estudios de la gestión privada del agua}

En lo que refiere al proceso de privatización, que se inició en América Latina a comienzos de la década del noventa, se advierte que existe gran cantidad de bibliografía que estudia este período, con énfasis principalmente en el rol que ejerció el Estado y en las consecuencias sociales de la privatización de estos servicios esenciales.

Ahora bien, ¿cuál es el fin o los fines que le han sido asignados al Estado en esta nueva etapa de privatización de los servicios públicos? Las reformas neoliberales impulsadas por el Consenso de Washington, que tuvieron lugar en América Latina a comienzos de los años noventa, implicaron la asunción de nuevos roles por parte del Estado: “...en lugar de producir directamente los bienes y los servicios, ahora intenta regular las condiciones de prestación de esas mismas funciones, que fueran transferidas a otros agentes sociales..." (Oszlak, Felder y Forcinito, 2000, p. 4). Para llevar a cabo este rol en Argentina se conformaron agencias estatales especializadas, los Entes de Regulación y Control.

El trabajo de Oszlak, et al. (2000) tiene como objetivo identificar el déficit de capacidad institucional que presentaron los entes reguladores en Argentina y nos ofrece un marco general sobre las distintas dimensiones e indicadores significativos para poder analizar el rol de regulación del estado durante la etapa de privatización. Analizan las capacidades institucionales teniendo en cuenta las dimensiones siguientes: (a) las reglas de juego formales e informales que gobiernan las interacciones entre los actores participantes; (b) las relaciones interinstitucionales que se establecen entre los diferentes actores involucrados en el proceso de regulación, prestación y uso de los servicios; (c) la organización interna y la asignación de funciones de los entes reguladores y; (d) la disponibilidad de recursos físicos y humanos. Luego del análisis de cada una de estas dimensiones, llegan a la conclusión de que los organismos reguladores presentaron un déficit de capacidad institucional para poder cumplir con su rol poniendo en riesgo el derecho de la ciudadanía de acceder a servicios públicos básicos y esenciales bajo los principios de regularidad, uniformidad, continuidad y universalidad (Oszlak et al., 2000). 
Dentro de este marco general, nos interesa destacar distintos estudios que se han enfocado en analizar el desempeño de las empresas privadas que asumieron la prestación de los SAS en las principales ciudades de Argentina. Entre estos trabajos podemos mencionar los de Roitman y Ferro (2003); Azpiazu, Catenazzi y Forcinito (2004); Muñoz (2004); Azpiazu, Schorr, Crenzel, Forte y Marín (2005); Pesce (2006, 2008) y Azpiazu (2010). Los casos que abordan estos autores son el de Aguas Argentinas S.A. (AASA), que obtuvo la concesión de los SAS en el Área Metropolitana de Buenos Aires (AMBA) durante el período 1993-2006; el de Aguas Provinciales de Santa Fe S.A. (APSFSA), que prestó los SAS en 15 localidades de la provincia de Santa Fe durante los años 1995-2006, y el de Aguas Cordobesas S.A. (ACSA), que presta el servicio de agua en la ciudad de Córdoba desde 1997 a la actualidad.

Los autores retoman algunas de las dimensiones para dar cuenta del déficit de capacidad institucional que mencionamos anteriormente. A su vez, enfatizan los negociados existentes entre las concesionarias privadas y el Estado, situación que permitió la obtención de rentas extraordinarias por parte de las empresas. Con relación a la dimensión de las reglas de juego, se abordan para cada uno de los casos los aspectos siguientes: cómo se conformaron los marcos normativos; las principales características de los contratos de concesión; las sucesivas modificaciones contractuales que tuvieron lugar; la situación más favorable para las empresas que habría resultado de las renegociaciones en detrimento de los derechos de los usuarios; la evolución de los esquemas tarifarios y el impacto que los aumentos progresivos de las tarifas representó en los gastos de los hogares de menor poder adquisitivo. En cuanto a la población que, por sus condiciones socioeconómicas, no podía afrontar el pago de la tarifa, y para evitar que estas sufrieran el corte del servicio, se establecieron en algunos casos subsidios especiales solventados por el Estado nacional y/o provincial. Las empresas privadas fueron reticentes a implementar un tipo de tarifa que establezca subsidios cruzados o a destinar fondos propios para cubrir parte del costo del servicio a las familias de menor poder adquisitivo.

Con relación a la dimensión de las relaciones interinstitucionales se puso énfasis en los vínculos que existieron entre los entes reguladores, las agencias del Poder Ejecutivo y la empresa privada. Se analizó cuál fue el grado de independencia con el que contaron los entes para poder llevar a cabo sus funciones y cuáles fueron los condicionamientos impuestos tanto por las agencias del Poder Ejecutivo como por los consorcios privados que limitaron el ejercicio de las funciones de los entes especializados. Los autores identifican que, en general, durante el proceso de privatización, se conformó un modelo de gestión que supuso una suerte de doble captura del regulador: por parte del interés privado y del poder político (Azpiazu et al., 2005, p. 50). 
En los distintos casos de estudio, los autores concluyen que, al analizar los resultados alcanzados por la gestión privada del servicio, se observó una gran disparidad entre las metas previstas y las efectivamente alcanzadas. Tanto en la firma de los contratos como en el diseño de los planes de mejora y expansión de los servicios, las empresas se comprometieron a alcanzar una serie de objetivos relativos a niveles de calidad (cortes del servicio, potabilidad del agua, dotación de producción, niveles de presión) y montos mínimos de inversión que debían realizarse en períodos determinados. Sin embargo, en el estudio de cada uno de los casos, se detectaron distintos incumplimientos, que contaron con la condescendencia oficial.

En el caso de Aguas Cordobesas S.A., el incumplimiento más grave tuvo que ver con la instalación de medidores. Para el año 2001 la prestadora debía tener colocados 81.000 medidores de los cuales solamente había instalado 31.000. Esto implicó sanciones por parte del Ente Regulador (Pesce, 2008, p. 54). Con relación al caso de Aguas Argentinas S.A., los principales incumplimientos que se detectaron tuvieron que ver con los niveles de inversión y con la ampliación de la cobertura. En cuanto a las inversiones previstas y efectivamente ejecutadas, durante el período 1993-2002, se detectó un incumplimiento de un 39\%. Esto se vio reflejado en el déficit de los niveles de cobertura ya que, para el mismo período, la empresa tenía el compromiso de alcanzar el $88 \%$ de hogares con acceso a agua potable y de $74 \%$ con acceso a desagües cloacales. Sin embargo, sólo alcanzó el $79 \%$ y $63 \%$ respectivamente (Azpiazu, 2010, pp. 6-7).

Por último, respecto a Aguas Provinciales de Santa Fe S.A., un informe publicado por el organismo regulador provincial demostró que la empresa cumplió con la inversión comprometida solamente en el primer año, pero en los años siguientes se produjo una disminución progresiva del monto invertido con respecto a los compromisos asumidos. En cuanto a la calidad del agua, dicho informe muestra que la empresa presentó un incumplimiento de un $95 \%$, al suministrar agua que no cumplía con los parámetros de calidad establecidos por la normativa vigente (ENRESS, 2004, citado por Muñoz, 2004).

A inicios del año 2002, mediante la sanción de la Ley de Emergencia Pública y Reforma del Régimen Cambiario (№ 25.561) se determinó el fin de la convertibilidad entre la moneda nacional y el dólar estadounidense. Esto dio inicio a un tramo de renegociación de los contratos entre los gobiernos y los consorcios privados, que tuvo como consecuencia la rescisión en los casos de ASSA y APSFSA y la vuelta de las empresas a manos estatales en 2006. En el caso de ACSA, en el mismo año, se produjo la salida del grupo francés Suez y la compra de las acciones por grupos nacionales, por lo tanto, la empresa sigue bajo gestión privada en la actualidad. Esta tendencia a la reestatización de los servicios dio lugar a la realización de nuevos estudios sobre las formas que adquiría el carácter público en la 
prestación de estos. En el siguiente apartado mencionamos algunos de los debates e investigaciones que se iniciaron en torno a estas cuestiones.

\subsection{Estudios de la gestión estatal del agua}

A inicios del Siglo XXI, se puede evidenciar una tendencia global a la reestatización de empresas proveedoras de agua potable y saneamiento que en las décadas previas habían sido privatizadas. En el año 2000 solamente dos empresas habían sido reestatizadas mientras que para el año 2015 se identificaron 235 casos en 37 países de todo el mundo (Kishimoto, Lobina y Petitjean, 2015).

En Argentina, en el período 1998-2015, once de las quince empresas de SAS que habían sido privatizadas fueron re-estatizadas. Esto generó una multiplicación de estudios que tuvieron como su principal eje analizar el nuevo rol asumido por el Estado en la prestación de estos servicios públicos esenciales. La literatura que aborda estos procesos reflexiona sobre qué tipo de gestión pública del servicio es la más adecuada para afrontar los desafíos actuales en el manejo del recurso y cuáles son las implicancias del retorno activo del Estado (en sus diferentes escalas) en la gestión de los SAS.

En Europa comenzó a surgir una nueva corriente que parte del concepto de Progressive Public Water Management (gestión pública progresista del agua). Las dimensiones que integran este concepto sintetizan los pilares para avanzar en una gestión pública progresista de los SAS. Estas son: (a) buena calidad del servicio; (b) universalización; (c) eficacia en la satisfacción de las necesidades y participación ciudadana; (d) reconocimiento del derecho humano al agua y saneamiento; (e) establecimiento de subsidios directos y cruzados de acuerdo al nivel socio-económico de los distintos grupos sociales y prohibición del corte del servicio por falta de pago; (f) sustentabilidad del servicio y gestión integrada del recurso hídrico; (g) buenas condiciones laborales y (h) existencia de una legislación progresista que regule y garantice los principios de la gestión pública (Hachfeld, Terhorst y Hoedeman, 2009, pp. 5-6).

Con relación a los estudios llevados a cabo en Argentina para analizar el comportamiento de las empresas reestatizadas contamos con los trabajos de Rocca (2008); Tobías (2014) y Tobías, Tagliavini y Orta (2017). Rocca (2008) aborda la capacidad regulatoria del Estado argentino en el servicio de agua potable y saneamiento en el AMBA. Esta área es provista por la empresa reestatizada Agua y Saneamientos Argentinos S.A. (AySA), quién asumió la prestación a partir de 2006, luego de la rescisión del contrato con la empresa privada AASA. Parte del enfoque ya mencionado en el trabajo de Oszlak et al. (2000), donde el accionar estatal en materia regulatoria es abordado a partir de la noción de capacidades estatales. La autora concluye que la capacidad regulatoria del estado durante 
el proceso de reestatización presentó más continuidades que rupturas con relación a la etapa de privatización. El hecho de que sea el mismo Estado nacional el encargado de prestar el servicio y, a su vez, de controlar y regular la prestación, requería que se dote al ente de control de autarquía y mayor autonomía para poder llevar a cabo con eficacia sus funciones, situación que no se dio en la práctica (Rocca, 2008).

Por otro lado, Tobías (2014) retoma la dimensión política del concepto de gobernabilidad, con énfasis en cómo entran en juego diversos actores con intereses, visiones y recursos particulares sobre el manejo del agua. Estudia de qué modo AySA interactúa con los actores involucrados en la gobernabilidad del agua y cuáles son las distintas miradas que existen en torno a la Gestión Integrada del Recurso Hídrico (GIRH). En su trabajo, argumenta que la reestatización del servicio de agua y saneamiento en el AMBA trajo aparejado un cambio en la concepción del recurso, dejando de lado la idea de mercancía propia del período privatizado, para ser interpretado como derecho humano. Este cambio de concepción se manifestó en la incorporación del derecho humano al agua dentro del marco regulatorio que rige a la empresa, en el establecimiento de subsidios tarifarios y en la inversión destinada a las grandes obras de infraestructuras tendientes a alcanzar la universalización del servicio. Sin embargo, argumenta la autora, quedan muchas cosas pendientes para alcanzar una gestión pública progresista del agua. Uno de los obstáculos está relacionado con que están presentes visiones distintas y hasta contrapuestas que los actores expertos poseen sobre la gestión del recurso, lo cual dificulta la implementación de políticas públicas progresistas (Tobías, 2014).

Por último, en el trabajo realizado por Tobías et al. (2017) se analizan algunas de las características que asume el nuevo modelo público del agua que se está desarrollando en Argentina, con énfasis en los casos de reestatización de AySA y Aguas Santafesinas S.A. (ASSA). Esta última, a partir de 2006, se hizo cargo de la provisión de SAS en la provincia de Santa Fe, luego de la rescisión del contrato con la empresa privada APSFSA. Los autores retoman las dimensiones comprendidas en el concepto de Progressive Public Water Management y analizan en qué medida las lógicas de gestión presentes en las empresas reestatizadas responden a los requisitos que hacen a una gestión progresista del agua.

Los estudios que hemos mencionado hasta el momento investigan los modos de gobernar los SAS y el déficit de capacidad institucional en las grandes ciudades y sus áreas metropolitanas donde, en general, los servicios fueron provistos por empresas privadas o estatales. Sin embargo, no son considerados en estos estudios los modos por medio de los cuales se abastecen de estos servicios en las áreas rurales donde la gestión estuvo a cargo de las propias comunidades que habitan estos territorios. A continuación, daremos cuenta de algunos estudios que abordan distintas experiencias de gestión comunitaria. 


\subsection{Estudios de la gestión comunitaria del agua}

Nos interesa explorar en este apartado los estudios que se focalizan en los sistemas de gestión comunitaria que surgieron en las áreas periféricas de las ciudades o en las zonas rurales. Estas áreas no son de interés de las empresas privadas debido a que la dispersión y menor cantidad de población hacen que la prestación del servicio no sea económicamente atractiva para organizaciones que buscan el lucro. En estas localidades son los propios vecinos, a partir de su capacidad de movilización y fuerza de trabajo, los que han tomado a su cargo la provisión de los SAS, asumiendo distintas formas organizativas según el área geográfica y las tradiciones socioculturales presentes en cada región. En este contexto, la gestión comunitaria es considerada como una respuesta espontánea y de acción colectiva para satisfacer las necesidades de abastecimiento de agua en una localidad, y puede entenderse como un modelo de gestión autónomo bajo el cual una comunidad se organiza y establece sus propias reglas para el aprovechamiento del recurso (Lockwood, 2002).

Las prestadoras comunitarias reciben distintas denominaciones según cada uno de los países y regiones en los que se crean: Comités Comunitarios de Agua en México, Cooperativas de Agua en Argentina, Juntas de Acción Comunal o Juntas Administradoras de Acueducto en Colombia, entre otras. Estas organizaciones son sujetos de control y vigilancia y deben cumplir con las mismas reglas que los grandes prestadores de servicios públicos. Sin embargo, cuentan con menos recursos que estas últimas para poder alcanzar esos objetivos.

Con relación a los estudios llevados a cabo para este tipo de gestión, podemos mencionar los de Allen, Dávila y Hofmann (2005); Sandoval (2011) y Roa, Brown y Roa (2015). Los autores abordan distintas formas de organización comunitaria del agua en México, Venezuela y Colombia, que se conformaron principalmente en las áreas rurales y/o en las zonas periféricas de las grandes ciudades. Las dimensiones que se tienen en cuentan para llevar a cabo estos estudios también se derivan, como en los casos anteriores, de los conceptos de gobernabilidad y capacidad institucional. En cuanto a gobernabilidad, encontramos algunos de los interrogantes propuestos por los autores mencionados anteriormente tales como: ¿Cómo se gestionan los servicios? ¿Cuál es la metodología utilizada para la toma de decisiones? ¿Cuáles son los fines y valores de políticas públicas que predominan entre los organismos responsables de la provisión y regulación de los SAS?

Por otro lado, en cuanto al concepto de capacidad institucional, los autores tienen en cuenta los aspectos siguientes: cómo se conforma la estructura administrativa; los modos de elección de las autoridades directivas; las reglas formales e informales que regulan su accionar; las reglas de acceso, uso y control del agua; las formas de participación comunitaria 
en los procesos de toma de decisiones; el nivel de acceso a la información y la relación que se establece entre los operadores comunitarios y el Estado en sus distintas escalas.

A partir de las dimensiones antes mencionadas, los autores establecen comparaciones entre la organización comunitaria y la organización estatal. Entre algunas de las conclusiones a las que arriban consideramos importante resaltar que las lógicas de gestión comunitaria se caracterizan por los aspectos siguientes: los usuarios participan en la planificación e implementación de proyectos; las autoridades de los operadores de agua se eligen en asambleas comunitarias por medio del voto y se busca garantizar el acceso al agua a toda la comunidad, al considerarlo un derecho colectivo. Las organizaciones pequeñas tienen mayores dificultades para acceder a las diversas fuentes de financiación y subsidios para realizar mejoras en su infraestructura, debido a que se les exigen los mismos requisitos que a los grandes operadores privados o estatales. Esto dificulta su posibilidad de atender las demandas de las comunidades abastecidas. Los autores se preocupan por dar cuenta de cómo se pueden mejorar las capacidades de las organizaciones comunitarias a partir de políticas públicas que tiendan a resolver las dificultades técnicas y económicas que deben enfrentar diariamente (Roa et al., 2015).

Por último, nos interesa hacer referencia a una serie de trabajos de investigación sobre experiencias comunitarias de gestión de agua realizados en el marco del proyecto "Democratización de la gobernabilidad de los servicios de agua y saneamiento mediante innovaciones sociotécnicas" ". Los casos estudiados fueron: (a) un sistema de potabilización de agua manejado por la comunidad implementado en el corregimiento de Mondomo, Colombia; (b) el diseño y ejecución con participación comunitaria de una planta de tratamiento de aguas residuales en la vereda La Vorágine, Colombia; (c) la implementación del Sistema Integrado de Saneamiento Rural (SISAR) en el estado de Ceará, Brasil y (d) un caso de gestión participativa en un sistema de tratamiento de agua en una comunidad quilombola de Minas Gerais, Brasil. Se evaluaron las experiencias de innovaciones socio-técnicas implementadas en cada uno de los casos, sus posibilidades de sustentabilidad en el tiempo, su replicabilidad en otros territorios y las implicancias de la participación comunitaria en la democratización del acceso y gestión de los SAS (García, Peña, Toro, Vargas, Cerón, Tamayo, Mena, Orjuela, Morales, Bolaños y Vidal, 2015; Peña, García, Toro, Vargas, Ceron, Mena y Orjuela, 2015; Pádua, Roncarati y Sullyvan, 2015; Martins, Castro, Pena, Rezende y Heller, 2015).

\footnotetext{
7 El proyecto fue financiado por el Séptimo Programa Marco de la Unión Europea para la investigación, el
} desarrollo tecnológico y la demonstración, mediante el contrato $N^{\circ} 320303$ (http://desafioglobal.org/es/). 
Para resumir los temas abordados en este apartado, presentamos la Tabla I en la cual se muestran los aspectos principales de cada una de las lógicas de gestión analizadas ${ }^{8}$.

Las desigualdades existentes que mencionamos han motivado acciones de protesta y lucha impulsadas tanto por ciudadanos de forma individual como por organizaciones sociales. A continuación, nos interesa destacar algunas investigaciones que se focalizan en analizar los conflictos y las luchas sociales por el agua, puesto que las consideramos fundamentales para poder entender y explicar cómo se van generando acciones que promueven una mayor democratización en el acceso y gestión de los SAS.

1 Estudios de los conflictos por el agua

El estudio de los conflictos por el agua está estrechamente vinculado con el modo en cómo se gobierna el recurso hídrico, pero incorpora la variable de las acciones llevadas a cabo por la sociedad civil. La vinculación con el tema anterior está dada también porque los cambios de las lógicas de gestión y el fortalecimiento de los derechos de ciudadanía no son decisiones que se toman de manera unidireccional desde el Estado, sino que, muchas veces, son impulsadas y promovidas por luchas sociales, tal como sostiene Castro (2007). El autor investiga cómo se han ido ampliando los derechos ciudadanos y democratizado la gestión y el acceso a estos servicios a partir de las luchas ciudadanas, las luchas por la inclusión y por la obtención de los derechos a una ciudad justa.

Tabla I. Lógicas de gestión de los SAS

8 Para la elaboración de la tabla se sigue la metodología que se presenta en un trabajo que evalúa la gestión del recurso hídrico a nivel de cuenca (Hordijk, Sara y Sutherland, 2014, p. 135). 


\begin{tabular}{|c|c|c|c|c|}
\hline \multirow[b]{2}{*}{ Dimensiones } & \multicolumn{4}{|c|}{ Lógica de Gestión } \\
\hline & Privatista* & $\begin{array}{l}\text { Estatal Racional } \\
\text { Administrativa* }\end{array}$ & Comunitaria $^{*}$ & $\begin{array}{l}\text { Gestión Público } \\
\text { Progresista** }\end{array}$ \\
\hline $\begin{array}{l}\text { Proceso de toma } \\
\text { de decisiones }\end{array}$ & $\begin{array}{r}\text { Jerárquica/verticalista/sin } \\
\text { participación ciudadana }\end{array}$ & $\begin{array}{r}\text { Jerárquica/Verticalista/partic } \\
\text { i-pación restringida }\end{array}$ & $\begin{array}{r}\text { Horizontal/participación } \\
\text { comunitaria }\end{array}$ & $\begin{array}{rr}\text { Horizontal/ } & \text { participación } \\
\text { ciudadana }\end{array}$ \\
\hline $\begin{array}{l}\text { Concepción del } \\
\text { acceso }\end{array}$ & $\begin{array}{r}\text { Mercantilización y exclusión, } \\
\text { acceden quienes pueden } \\
\text { pagar }\end{array}$ & $\begin{array}{r}\text { Derecho social, servicio } \\
\text { público, acceso } \\
\text { independiente de las } \\
\text { condiciones sociales y } \\
\text { económicas }\end{array}$ & $\begin{array}{r}\text { Derecho colectivo, } \\
\text { determinado por la } \\
\text { pertenencia a la comunidad }\end{array}$ & Derecho humano \\
\hline $\begin{array}{l}\text { Principio que } \\
\text { guía la expansión } \\
\text { de los SAS }\end{array}$ & $\begin{array}{r}\text { Rentabilidad/inversión } \\
\text { privada en áreas rentables }\end{array}$ & $\begin{array}{r}\text { Inversión público- } \\
\text { estatal/universalización del } \\
\text { acceso }\end{array}$ & $\begin{array}{r}\text { inversión con fondos } \\
\text { provenientes de los socios/ } \\
\text { garantizar el acceso a todos } \\
\text { los miembros de la } \\
\text { comunidad }\end{array}$ & $\begin{array}{r}\text { Inversión público- } \\
\text { estatal/universalización del } \\
\text { acceso }\end{array}$ \\
\hline $\begin{array}{l}\text { Principales } \\
\text { actores }\end{array}$ & Sector privado & $\begin{array}{r}\text { Políticos profesionales y } \\
\text { expertos técnicos }\end{array}$ & $\begin{array}{r}\text { Vecinos, organizaciones } \\
\text { comunitarias }\end{array}$ & $\begin{array}{r}\text { Actores } \\
\text { comunitarios/organizaciones } \\
\text { sociales/funcionarios } \\
\text { políticos }\end{array}$ \\
\hline $\begin{array}{l}\text { Subsidios } \\
\text { tarifarios }\end{array}$ & $\begin{array}{r}\text { Sin subsidios por parte de } \\
\text { la empresa/cortes del } \\
\text { servicio } \\
\text { permitidos/subsidios } \\
\text { especiales a cargo del } \\
\text { Estado nacional y/o } \\
\text { provincial }\end{array}$ & $\begin{array}{r}\text { Subsidios } \\
\text { directos/subsidios } \\
\text { cruzados/subsidios del } \\
\text { Estado nacional y/o } \\
\text { provincial }\end{array}$ & $\begin{array}{r}\text { Subsidios directos por parte } \\
\text { de la asociación } \\
\text { comunitaria/Subsidios } \\
\text { cruzados/subsidios } \\
\text { ocasionales del Estado } \\
\text { nacional y/o provincial }\end{array}$ & $\begin{array}{r}\text { Subsidios directos/subsidios } \\
\text { cruzados/subsidios del } \\
\text { Estado nacional y/o provincial }\end{array}$ \\
\hline
\end{tabular}

*Lógica existente.

**Lógica deseada.

Fuente: elaboración propia sobre la base de la bibliografía citada up supra.

Entre los trabajos que analizan distintos conflictos por el agua que tuvieron lugar en América Latina podemos mencionar los de Castro, Kloster y Torregrosa (2004); Kloster y De Alba (2007); Castro (2007) y Kloster (2016). Los conflictos que registran los autores asumen distintas formas tales como: demandas pacíficas dirigidas a las autoridades a fin de obtener acceso a agua potable, acciones violentas que involucran la destrucción de infraestructura para el suministro de agua, confrontaciones armadas entre las fuerzas de seguridad y miembros de la población, entre otras. Asimismo, establecen una serie de dimensiones para poder estudiar los conflictos por el agua. Estas son: (a) nivel y tipo de organización de los actores que realizan las acciones; (b) tipos de actores a los cuales se dirigen las acciones; (c) razones alegadas por los protagonistas para realizar sus acciones; (d) instrumentos usados en las acciones y (e) resultados y efectos de las acciones.

Los autores concluyen que el aumento de los conflictos por el agua está vinculado con la profundización de las desigualdades económicas, sociales, culturales, políticas y territoriales. Demuestran que no es posible explicar esos conflictos sólo por factores 
naturales, técnicos o demográficos tales como, la escasa disponibilidad de agua, la aridez o la presión del crecimiento demográfico y urbano. Por el contrario, las raíces del problema están vinculadas con la existencia y reproducción de desigualdades socioeconómicas y políticas que determinan la exclusión de grandes sectores de la población del acceso a estos servicios y bienes esenciales (Castro et al., 2004, p. 340).

\section{Situación en la provincia de Santa Fe}

La provincia de Santa Fe (Argentina) tiene una superficie de más de $130 \mathrm{mil} \mathrm{km}^{2}$ y una población de más de 3 millones de habitantes. Está dividida política y administrativamente en diecinueve departamentos que a su vez se subdividen en 362 distritos. De estos distritos, 50 son municipios y 312 asumen la forma de comunas. Según el Censo Nacional de Población, Hogares y Viviendas (CNPHV) realizado por el Instituto Nacional de Estadísticas y Censos (INDEC) en el año 2010, el 82\% de la población santafesina tenía acceso a agua potable, mientras que sólo un $45 \%$ de la población tenía acceso a desagüe cloacal en condiciones seguras.

La realidad del territorio santafesino se caracteriza por la presencia de una gran variedad de formas de gestión del agua que se fueron constituyendo a lo largo de un proceso histórico. De las 362 localidades que integran la provincia solamente $15^{9}$ son abastecidas por la empresa estatal ASSA, mientras que las restantes localidades deben prestarse por sí mismas los servicios. A su vez, la empresa de agua y saneamiento perteneciente al Estado provincial cuentan con subsidios que alcanzan a cubrir el $40 \%$ de sus gastos operativos mientras que los operadores de las localidades restantes no cuentan con ningún tipo de subsidio. Esto genera fuertes inequidades en términos de las capacidades institucionales con las que cuentan los pequeños y medianos prestadores para hacer frente a las desigualdades en el acceso a los SAS en los territorios que están a su cargo.

Entre estos pequeños y medianos prestadores están presentes en la provincia 108 cooperativas de agua y saneamiento que nacieron mediante la iniciativa de los vecinos de las localidades rurales, que no contaban con los servicios básicos. Si bien, a partir de que se inicia el proceso de reestatización de los servicios en el año 2006, se emprendieron acciones tendientes a abordar distintos aspectos relativos al problema del agua y saneamiento, los programas implementados no fueron suficientes para brindar una solución integral, definitiva y concluyente. En trabajos previos, se consideró que la persistencia de esta cuestión está relacionada con la existencia de una lógica de gestión rígida de los SAS que, a pesar de los reclamos persistentes de la ciudadanía, organismos de defensa de los usuarios y consumidores, cooperativas de agua y sus asociaciones federativas, académicos, agencias

9 Estas 15 localidades son parte del grupo de las ciudades más grandes de la provincia (más de 20.000 habitantes) y representan aproximadamente el $60 \%$ de la población. 
de investigación nacionales, intendentes y concejales, continúa configurada por un marco normativo elaborado durante el proceso privatizador (Orta, Antón, Portapila y Forte, 2016).

\section{Reflexiones finales}

Las nociones e ideas desarrolladas hasta aquí constituyen herramientas conceptuales fundamentales para poder interrogarnos acerca de cuáles son los desafíos que enfrentan los distintos prestadores de los SAS en la provincia de Santa Fe. Por las cuestiones mencionadas pensamos que los interrogantes, conceptos y dimensiones de análisis que nos ofrece la literatura que revisamos a lo largo del trabajo son pertinentes para poder abordar las cuestiones siguientes: ¿Cómo se gobernaron los servicios de agua en Santa Fe a lo largo de la historia? ¿Cuáles son las consecuencias de los distintos principios de gobernabilidad que predominaron en el proceso histórico de desarrollo de los SAS sobre la democratización en el acceso y gestión de estos servicios vitales? ¿Cuáles son las capacidades institucionales con las que cuentan los distintos prestadores de los servicios? ¿Cuáles son los conflictos y luchas por el agua que se dieron en la provincia en los últimos tiempos? y ¿Cuáles fueron los efectos y resultados de estas luchas y conflictos en el diseño de las políticas públicas relacionadas con la gestión del recurso hídrico?

Como pudimos observar a lo largo del trabajo, los principios que caracterizan a cada una de las lógicas de gestión de los SAS tienen implicancias directas sobre la profundización o superación de las desigualdades en el acceso y gestión de estos servicios. El predominio de la lógica estatista racional administrativa durante la mayor parte del Siglo XX posibilitó un avance sustancial en el proceso de universalización del acceso a estos servicios públicos, pero no garantizó una ampliación de la democratización en la gestión debido a que los procesos de toma de decisiones involucraban principalmente a los políticos profesionales y a los expertos técnicos. El regreso de la lógica de gestión privatista en la década del 90 en Argentina profundizó las desigualdades en el acceso y gestión de los SAS, al limitar las capacidades del Estado para ejercer su rol de control y regulador de las empresas privatizadas. Las lógicas de gestión comunitaria existentes en América Latina presentan lecciones de cómo una forma de organización de los SAS, con participación de todos sus miembros, posibilita un avance hacia la democratización, más allá de la situación desigual en la que se encuentran estos prestadores, con relación a las empresas estatales o privadas.

Por último, consideramos que el escenario que se abre a partir de la etapa de la reestatización de los servicios y los criterios que nos ofrece el enfoque de la gestión pública y progresista del agua nos posibilita cuestionarnos sobre cuáles deben ser los aspectos que debe asumir una gestión pública de los SAS para poder garantizar así el derecho humano de acceso a agua y saneamiento en todo el territorio provincial. 


\section{Referencias bibliográficas}

Azpiazu, D. (2010). Privatización del agua y el saneamiento en Argentina: El caso paradigmático de Aguas Argentinas S.A. Revista Vértigo -la revue électronique en sciences de l'environnement. Serie $N^{\circ}$ 7, 1-11. Recuperado de: http://vertigo.revues.org/9730\#text

Azpiazu, D., Catenazzi, A. y Forcinito, K. (2004). Recursos públicos, negocios privados: agua potable y saneamiento ambiental en el Área Metropolitana de Buenos Aires. Buenos Aires: Universidad Nacional de General Sarmiento.

Azpiazu, D., Schorr, M., Crenzel, E., Forte, G., y Marín, J. C. (2005). Agua potable y saneamiento en Argentina. Privatizaciones, crisis, inequidades e incertidumbre futura. Cuadernos del CENDES, (59), 45-67.

Castro, J. E. (2007). El estudio interdisciplinario de los conflictos por el agua en el medio urbano: una contribución desde la sociología. Cuadernos del CENDES, $N^{\circ} 66,21$ 46.

Castro, J. E. (2011). El proceso de democratización de la gestión de servicios públicos esenciales de agua y saneamiento. Hábitat y Sociedad, $N^{\circ} 2,13-37$.

Castro, E. (2016a). Desigualdad estructural y determinación social. Waterlat-Gobacit Network Working Papers, Vol. $3 N^{\circ}$ 9, 8-29. Recuperado de: http://waterlat.org/WPapers/WPSATGSA39.pdf

Castro, E. (2016b). Socio-technical solutions for the provision of safe water and sanitation services in vulnerable communities: a synthesis. Waterlat-Gobacit Network Working Papers, Vol. $2 \quad N^{\circ}$ 16, 7-39. Recuperado de: http://waterlat.org/WPapers/WPSPIDES216.pdf

Castro, J. E., Kloster, K., y Torregrosa, M. L. (2004). Ciudadanía y gobernabilidad en México: el caso de la conflictividad y la participación social en torno a la gestión del agua. En B. Marín (Ed.) El Agua en México Visto desde la Academia, (pp. 199-232). México, DF: Academia Mexicana de Ciencias.

García, M; Peña, M; Toro, A; Vargas, J; Cerón, V; Tamayo, S; Mena, E; Orjuela, V; Morales, D; Bolaños, S. y Vidal, R. (2015). Gestión comunitaria del agua rural en Colombia y asociatividad comunitaria. Waterlat-Gobacit Network Working Papers, Vol. $2 N^{\circ}$ 11, 14-112. Recuperado a partir de: http://waterlat.org/WPapers/WPSPIDES211.pdf

Goldman, M. (2007). How 'Water for All!' policy became hegemonic: The power of the World Bank and its transnational policy networks, Geoforum Vol. 38, N $5,786-800$. 
Hachfeld, D., Terhorst, P. y Hoedeman, O. (2009). Progressive public water management in Europe. In search of exemplary cases (Reclaming Public Water discussion paper). Transnational Institute and Corporate Europe Observatory, 1-17

Hanf, K. y Jansen, A.-I. (1998). Governance and Environment in Western Europe: Politics, Policy and Administration. Harlow: Addison Wesley Longman.

Harvey, D. (2005). The 'New' Imperialism: Accumulation by Dispossession. Socialist Register, Vol. 40, 63-87.

Hordijk, M., Sara, L. M., y Sutherland, C. (2014). Resilience, Transition or Transformation? A Comparative Analysis of Changing Water Governance Systems in Four Southern Cities. Environment and Urbanization, Vol. 26, $N^{\circ} 1,130-146$.

Justo, J. B. (2013). El derecho humano al agua y al saneamiento frente a los Objetivos de Desarrollo del Milenio (ODM) (Colección Documentos de Proyectos). Santiago de Chile: ONU-CEPAL.

Kishimoto, S., Lobina, E. y Petitjean, O. (2015). Our Public Water Future: The Global Experience With Remunicipalisation. Amsterdam: Transnational Institute.

Kloster, K. (2016). Las Luchas por el Agua en México 1990-2010. México DF: Universidad Autónoma de la Ciudad de México.

Kloster, K. y De Alba, F. (2007). El agua en la ciudad de México y el factor de fragmentación política. Revista Perfiles Latinoamericanos, Vol. 14, N²9, 137-159.

Lockwood, H. (2002). Mecanismos de apoyo institucional para los sistemas rurales de agua potable y saneamiento manejados por las comunidades en América Latina (Environmental Health Project). Washington, D.C: Agencia para el Desarrollo Internacional de los Estados Unidos. Recuperado a partir de: http://www.ehproject.org/PDF/Strategic_papers/EHP\%20SR-6SPANISH.pdf

Martins, E; Castro, S; Pena, J; Rezende, S. y Heller, L. (2015). Evaluación político-institucional del Modelo SISAR en el nordeste de Brasil. Waterlat-Gobacit Network Working Papers, Vol. $2 N^{\circ}$ 6, 20-308. Recuperado a partir de: http://waterlat.org/WPapers/WPSPIDES26.pdf

McDonald, D. (Ed.). (2015). Servicios Públicos en el sur global. Mirada crítica a nuevas formas de gestión. Madrid: Clave intelectual.

Mora, J. y Dubois, V. (2015). Implementación del derecho humano al agua en América Latina. Caracas: CAF.

Muñoz, A. D. (2004). Análisis del Informe sobre cumplimiento de obligaciones contractuales de Aguas Provinciales compañía SUEZ en Santa Fe Argentina. Santa Fe: 
Asamblea Provincial por el Derecho al Agua. Recuperado a partir de: http://tallerecologista.org.ar/menu/archivos/IncumplimientosSuez.pdf

Orta, M., Antón, G., Portapila, M., y Forte, G. (2016). La calidad del agua para consumo humano como problemática social y política en Santa Fe, 2007-2015. Ponencia presentada en el $3^{\circ}$ Encuentro de Investigadores en Formación en Recursos Hídricos, Ezeiza: Instituto Nacional del Agua. Recuperado de: https://www.ina.gov.ar/ifrh-2016/trabajos/IFRH_2016_paper_54.pdf

Oszlak, O., Felder, R. y Forcinito, K. (2000). La capacidad regulatoria del Estado en Argentina. Buenos Aires: Facultad de Ciencias Económicas-Universidad de Buenos Aires. Área de Investigación de Privatización y Regulación.

Pádua, V; Roncarati, D. y Sullyvan, A. (2015). Generación participativa de un sistema de tratamiento de agua en una comunidad quilombola en Minas Gerais, Brasil. Waterlat-Gobacit Network Working Papers, Vol. $2 N^{\circ}$ 12, 14-103. Recuperado a partir de: http://waterlat.org/WPapers/WPSPIDES212.pdf

Peña, M; García, M; Toro, A; Vargas, J; Ceron, V; Mena, E. y Orjuela, V. (2015). Empoderamiento, resiliencia y sustentabilidad: evaluación de un sistema integrado de agua y saneamiento en la comunidad rural de La Vorágine, Colombia. WaterlatGobacit Network Working Papers, Vol. 2 N 9, 15-141. Recuperado a partir de: http://waterlat.org/WPapers/WPSPIDES29.pdf

Pesce, J. (2006). La concesión del servicio de agua potable y saneamiento en la provincia Santa Fe (Vol. 15). Buenos Aires: FLACSO, Área de Economía y Tecnología.

Pesce, J. (2008). La concesión del servicio de agua potable al capital privado en la ciudad de Córdoba: Una experiencia conflictiva, 1997-2006. Buenos Aires: FLACSO.

Roa, M. C. R., Brown, S. y Roa, C. E. R. (2015). Jerarquía de vulnerabilidades de las organizaciones comunitarias de agua en Colombia. Gestión y Ambiente, Vol. $18 \mathrm{~N}^{\circ}$ $2,51-79$

Rocca, M. (2008). Algo nuevo, algo usado, algo prestado... La reestatización del servicio de agua potable y saneamiento del Área Metropolitana de Buenos Aires (Documentos de Investigación Social No. 1). Buenos Aires: IDAES.

Roitman, M., y Ferro, G. (2003). La concesión de Aguas Provinciales de Santa Fe. Buenos Aires: CEER-UADE.

Sandoval, A. (2011). Entre el manejo comunitario y gubernamental del agua en la Ciénega de Chapala, Michoacán, México. Agricultura, sociedad y desarrollo, Vol. $8 N^{\circ} 3,367-$ 385. 
Spronk, S. J. (2007). The Politics of Water Privatization in the Third World, Review of Radical Political Economics, Vol. 39, $N^{\circ} 1,126-131$.

Swyngedouw, E. (2004). Social power and the urbanization of water: flows of power. Oxford: Oxford University Press.

Swyngedouw, E. (2005). Dispossessing H2O: the contested terrain of water privatization. Capitalism Nature Socialism, Vol. 16, No 1, 81-98.

Tobías, M. (2014). La re-estatización del servicio de agua y saneamiento y la gobernabilidad del agua en el Área Metropolitana de Buenos Aires (2006-2012) (Tesis de Maestría). Buenos Aires: Universidad Nacional de San Martín (UNSAM).

Tobías, M; Tagliavini, D. y Orta, M. (2017). El auge de las re-estatizaciones en Argentina ¿Hacia un nuevo modelo público del agua? Waterlat-Gobacit Network Working Papers, Vol. $4 N^{\circ}$ 2, 78-118. Recuperado a partir de: http://waterlat.org/WPapers/WGWPVol4No2.pdf

\section{Otros documentos consultados}

ONU y UNESCO. (2003). Informe de las Naciones Unidas sobre el Desarrollo de los Recursos Hídricos en el Mundo: Agua para todos, agua para la vida. París, Nueva York y Oxford: UNESCO y Berghahn Books.

OMS y UNICEF (2004). Alcanzar los ODM en materia de agua potable y saneamiento (Meta $10 \mathrm{del}$ ODM 7) Evaluación a mitad de período de los progresos realizados. Nueva York: OMS-UNICEF.

OMS y UNICEF (2007). La meta de los ODM relativa al agua potable y el saneamiento: el reto del decenio para zonas urbanas y rurales. Ginebra: OMS-UNICEF.

OMS y UNICEF (2014). Progresos en materia de agua potable y saneamiento: informe de actualización 2014. Nueva York: OMS-UNICEF.

OMS y UNICEF. (2015). Argentina: Estimates on The Use of Water Sources and Sanitation Facilities (1980-2015). Nueva York: OMS-UNICEF.

OMS y UNICEF (2016). Desigualdades en materia de saneamiento y agua potable en América Latina y el Caribe. Nueva York: OMS-UNICEF.

ONU. (2000). Declaración del Milenio. Resolución aprobada por la Asamblea General de Naciones Unidas. Nueva York: ONU.

ONU. (2010). El progreso de América Latina y el Caribe hacia los Objetivos de Desarrollo del Milenio. Desafíos para lograrlos con igualdad. Santiago de Chile: Comisión Económica para América Latina y el Caribe (CEPAL). 
ONU (2015). Transformar nuestro mundo: la agenda 2030 para el Desarrollo Sostenible. Nueva York: ONU. 